\title{
Overcoming Data Challenges Examining Oral Health Disparities in Appalachia
}

\author{
Denise D. Krause ${ }^{1}$, Warren L. May ${ }^{1}$, Jeralynne S. Cossman ${ }^{2}$ \\ ${ }^{1}$ University of Mississippi Medical Center \\ ${ }^{2}$ Mississippi State University
}

\begin{abstract}
Objective: The objective of our study of oral health disparities in Appalachia was to use existing data sources to geographically analyze suspected disparities in oral health status in the 420 counties of Appalachia, and to make sub-state comparisons within Appalachia and to the rest of the nation. The purpose of this manuscript is to describe the methods used to overcome challenges associated with using limited oral health data to make inferences about oral health status.
\end{abstract}

Methods: Oral health data were obtained from the Behavioral Risk Factor Surveillance System (BRFSS). Because the BRFSS was designed for state-level analysis, there were inadequate numbers of responses to study Appalachia by county. We set out to determine the smallest possible unit we could use, aggregating data to satisfy CDC minimum requirements for spatially identified responses. For sub-state comparisons, data were first aggregated to Appalachian and non-Appalachian regions within Appalachian states. Next, urban versus rural areas within Appalachian and non-Appalachian regions were examined. Beale codes were used to define metropolitan and non-metropolitan statistical regions for the United States.

Results: Aggregating the data as described proved useful for smoothing the data used to analyze oral health disparities, while still revealing important sub-state differences. Using geographic information systems to map data throughout the process was very useful for determining an effective approach for our analysis.

Discussion: Studying oral health disparities on a regional or national level is difficult given a lack of appropriate data. The BRFSS can be adapted for this purpose; however, there is a limited number of oral health questions and because they are also optional, they are not routinely asked by all states. Expanding the BRFSS to include a larger sampling frame would be very helpful for studying oral health disparities.

Conclusions: Novel techniques were introduced to use BRFSS data to study oral health disparities in Appalachia, which provided informative sub-state results, useful to health planners for targeting intervention strategies.

Keywords: Behavioral Risk Factor Surveillance System (BRFSS), Beale codes, oral health, disparities, Geographic Information Systems (GIS) 


\section{Introduction}

The Surgeon General's report "Oral Health in America" declared that no less than a "silent epidemic of oral disease is affecting our most vulnerable citizens - poor children, the elderly, and many members of racial and ethnic minority groups."1 There are many factors which affect equitable access to oral health care. Understanding those inequities is a prerequisite to eliminating them. For example, socioeconomic status is a key factor contributing to oral health disparities among population subgroups in the U.S. People living in rural areas also experience oral health disparities disproportionately.

Despite improved care over the last 20 years $^{2}$, dental care is still identified as the primary health need of U.S. children. ${ }^{3}$ Due to lack of care and inadequate preventive measures, childhood caries (also known as tooth decay) are the most common chronic disease among children - in fact, it is five to eight times more common than asthma. ${ }^{4}$ Even more alarming is the concentration of childhood caries: $80 \%$ of dental caries are found in $25 \%$ of children, most of whom are from lower socioeconomic households. ${ }^{5}$

Appalachia is a rural region known to be economically disadvantaged. As with other health indicators, oral health care exhibits disparities within the region. Funding was provided by the Appalachian Regional Commission to use existing data sources to geographically analyze suspected disparities in oral health status in the 420 counties that make up Appalachia, and to make sub-state comparisons within Appalachia, and to the rest of the nation. As a part of the overall study, we also examined relationships between oral health disparities, socioeconomic status indicators, and other indicators in that area. Details of the comprehensive analysis are reported elsewhere. ${ }^{6}$ Unfortunately, there is a paucity of available data relating to oral health status, in Appalachia, or the nation as a whole. We describe the methodology used to overcome challenges associated with limited availability of oral health data in an effort to make informed inferences about oral health status in the Appalachian region. Geographic visualization techniques were used to assess the usefulness of the oral health data throughout the project and to define a strategy for producing useful results. 


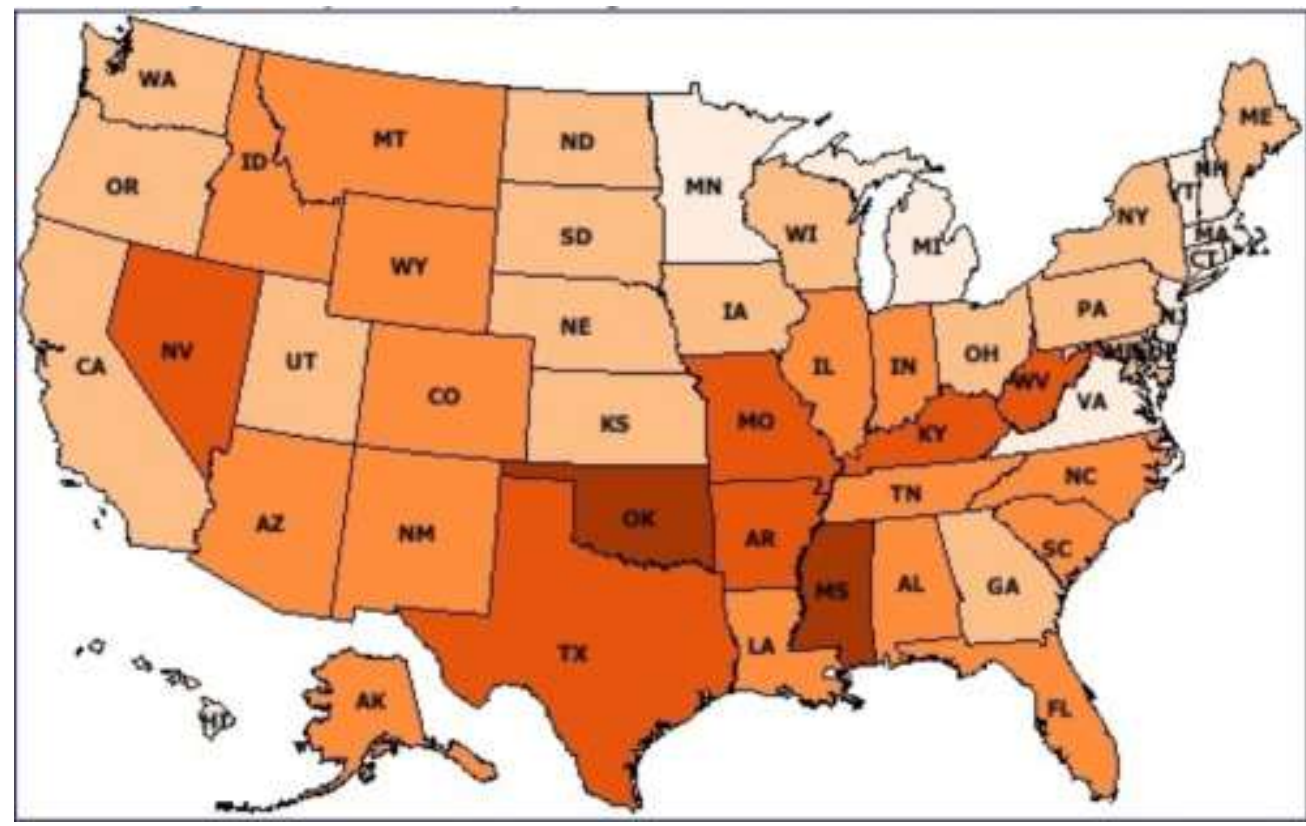

Dental Visit Within Past Year

$\square<=25 \%$

$25.1 \%$ to $30 \%$

$30.1 \%$ to $35 \%$

$35.1 \%$ to $40 \%$

$<=40.1 \%$

No Data

Figure 1. Percentage of respondents to the Behavioral Risk Factor Surveillance System survey reporting 'No' to having had a dental visit within the past year. BRFSS data are presented at the state level. Sources: Centers for Disease Control, U.S. Department of Health and Human Services, 2008.

\section{Methods}

The only known publicly available and readily accessible data source we could obtain to test sub-state oral health disparities was the Behavioral Risk Factor Surveillance System (BRFSS). The BRFSS survey is a continuous telephone health survey system which is used for monitoring health conditions and health-risk behaviors across the United States. The BRFSS provides highquality state-level data that informs policymakers of regional disparities in both health conditions and positive health practices (Figure 1). This survey, however, was not intended to be used for small area, or sub-state, estimates. Recently, there has been attention placed on developing statistical methodology using BRFSS data for small area estimation, such as health district or county-level analyses. ${ }^{7-12}$

The topic of oral health presents a special challenge. Unfortunately, there are only a few common questions pertaining to oral health included in the BRFSS survey ${ }^{13}$. Oral health questions are:

1. How long has it been since you last visited a dentist or dental clinic for any reason?

2. How many of your permanent teeth have been removed because of tooth decay or gum disease? Do not include teeth lost for other reasons, such as injury or orthodontics.

3. How long has it been since you had your teeth cleaned by a dentist or dental hygienist?

Additionally, these oral health questions have been included only as an optional module of the 
survey and have not been asked every year in every state, due to the overall length and expense of the survey. It is left to the discretion of individual states to decide whether to use the optional modules in any given year, and then they must have a budget to cover the additional expense. This can be especially problematic for poorer states, such as some of those in Appalachia. Ultimately, this leads to difficulty obtaining large enough sample sizes for annual county-level analysis. The Centers for Disease Control (CDC) requires at least 50 responses per county to use that question's responses for any given survey year. That means that very high response rates may be necessary in some rural counties for their data to be usable.

To examine the oral health data at the county level, we merged county identifiers obtained from the CDC with BRFSS responses. To aid in addressing the small sample limitation, we combined eight years (1999-2006) of data from the BRFSS to increase sample sizes at the county level. We used the survey question inquiring as to whether a person had a dental visit in the last 12 months. Additionally, we used the BRFSS coding scheme for the oral health indicator reporting the number of teeth that have been removed and imputed age, to recode variables as "Any teeth removed for ages 35-44", "Six or more teeth removed for ages 35-44", and "All teeth removed for age 65 and over". All three of these indicators refer to tooth loss attributed to decay or gum disease, not to injury or orthopedic treatment. This provided information on adult oral health status (any tooth loss or significant tooth loss), and senior oral health status (complete tooth loss). Participants with missing data (coded "9" or system missing) were eliminated. Raw proportions were examined for trends that would preclude using the combined estimates. SAS v 9.1.3 was used to combine the data, and the survey procedures in SAS were used to find countylevel estimates by including the CDC final weights ${ }^{14}$ and pre-defined strata in the estimation process.

A geographic information system (GIS) was built with sociodemographic and oral health data, and these data were mapped using ESRI ArcGIS 9.3 software. For the purposes of this paper, we illustrate the methodology by presenting maps pertaining to only one of the three oral health variables used for the overall study (dental visit in the past year). The dental visit variable has far more responses than the other oral health variables. Even using the variable with the greatest number of responses, we faced sample size limitations for county-level analysis. These challenges were greatly exacerbated for the other oral oral health variables on tooth loss.

Our original intention was to attain adequate sample sizes to estimate prevalence at the county level after combining multiple years of BRFSS data. By mapping oral health indicators at the county level, it became evident that geographic areas larger than counties would have to be considered to obtain sufficient sample sizes. County-level estimates were not feasible according to the CDC guidelines, even after combining several years of survey data, as many counties still had fewer than 50 respondents (Figure 2). As shown in Figure 2, many of the more rural counties and those states that did not participate in collecting oral health data in optional years proved to be most difficult in obtaining a clear picture of regional oral health.

At this point, the only sub-state comparison we could make using oral health data with more than 50 respondents was a comparison of Appalachian regions to non-Appalachian regions, within Appalachian and non-Appalachian states. The 420 counties of Appalachia were identified using 
designations established by the Appalachian Regional Commission. Again, estimates of prevalence for counties with 50 or more responses were mapped. This time counties with fewer than 50 were classified with the rest of the Appalachian or non-Appalachian region of the state (Figure 3) in an effort to provide at least some information for those counties that could not be shown due to limitations of sample size. This provided a more local view of some areas where data were available, but proved to be somewhat difficult to interpret.

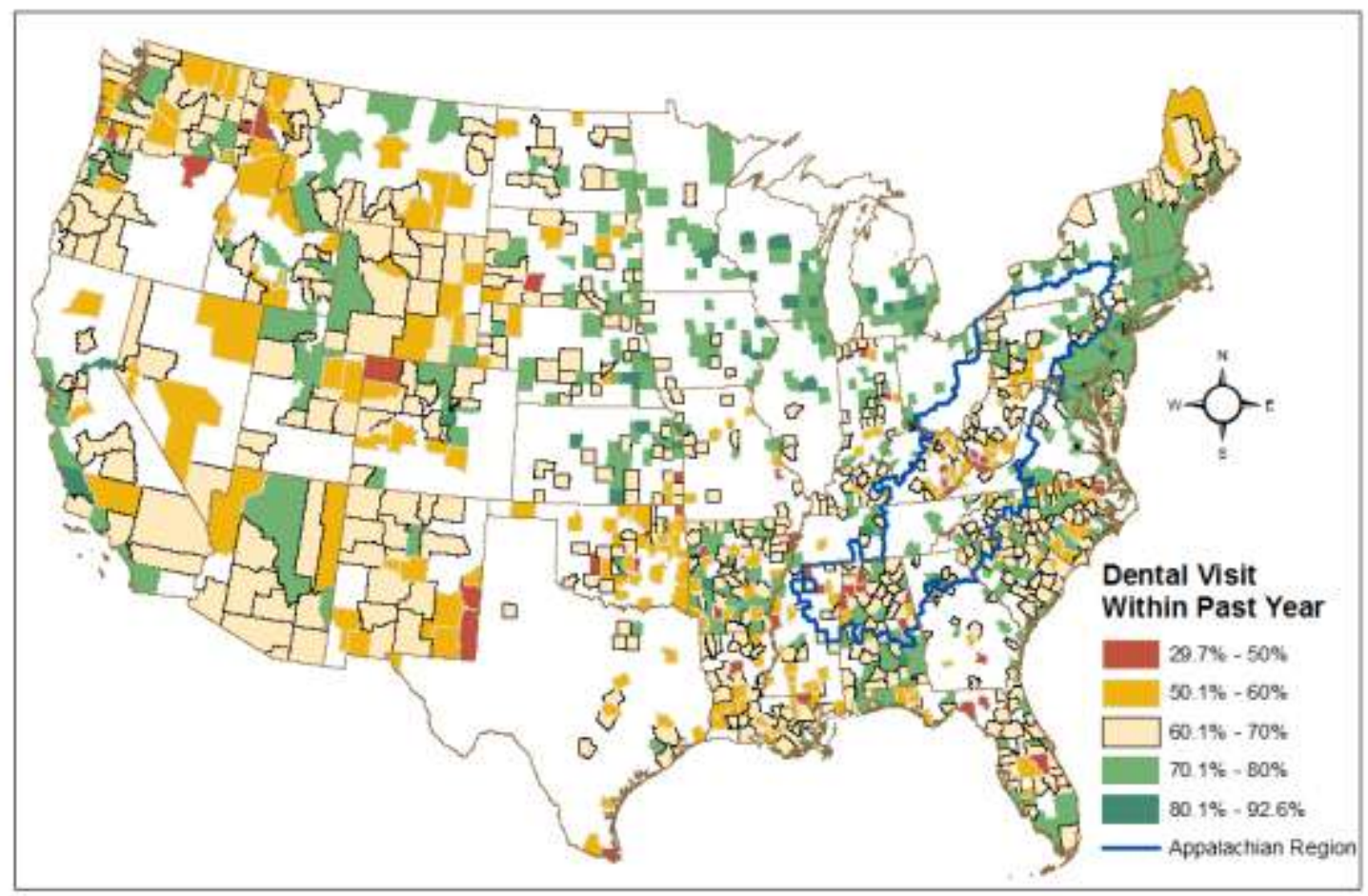

Figure 2. BRFSS data (1999-2006) mapped showing respondents of all ages who had a dental visit within the past year, for all U.S. counties. Even after combining eight years of data, many counties had inadequate number of responses to be included in the analysis. The Appalachian region outlined

in

blue. 


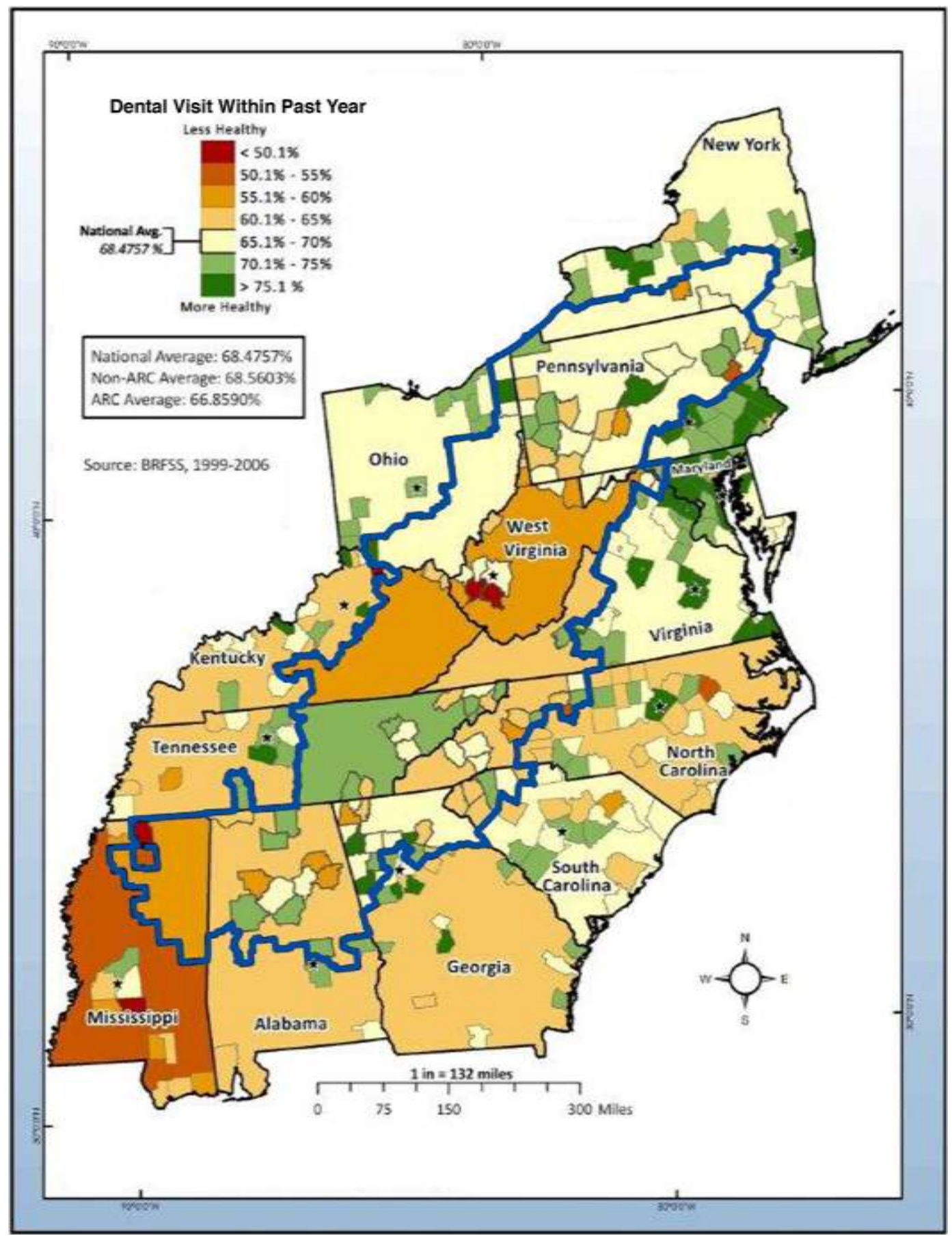

Figure 3. BRFSS oral health indicator for having had a dental visit within the past year, aggregated to obtain 50 or more responses. Some counties have an adequate number of responses. Others are aggregated to the Appalachian / non-Appalachian portions of the states. The Appalachian region is outlined in blue.

To more clearly represent these data and further expand our analysis, we set out to determine the smallest possible unit we could use, aggregating BRFSS data to satisfy CDC minimum 
requirements. Because we suspected that dental services vary depending on urban or rural settings, we decided to examine metropolitan versus non-metropolitan areas within Appalachian and non-Appalachian regions. Beale codes, developed by the U.S. Department of Agriculture ${ }^{15}$, were used to define the metropolitan and non-metropolitan statistical regions for sub-state comparisons within the Appalachian region and across the United States. Beale codes form a classification scheme that distinguishes metropolitan counties by size and non-metropolitan counties by degree of urbanization and proximity to metropolitan areas. Instead of typical ruralurban classifications based on population density, Beale codes account for proximity to metropolitan areas and, therefore, to potentially greater access to care. ${ }^{15}$

Each survey response that reported county of residence was assigned a Beale code and an Appalachian code. Again, due to sample size issues, we combined the nine categories of the original Beale classifications so that codes 1-3 represent metropolitan areas and codes 4-9 represent non-metropolitan areas. These designations are shown in Figure 4. The prevalence for each oral health indicator was then calculated for each of the following groups: (1) Appalachian/ metropolitan, (2) Appalachian/non-metropolitan, (3) non-Appalachian/metropolitan, and (4) nonAppalachian/non-metropolitan.

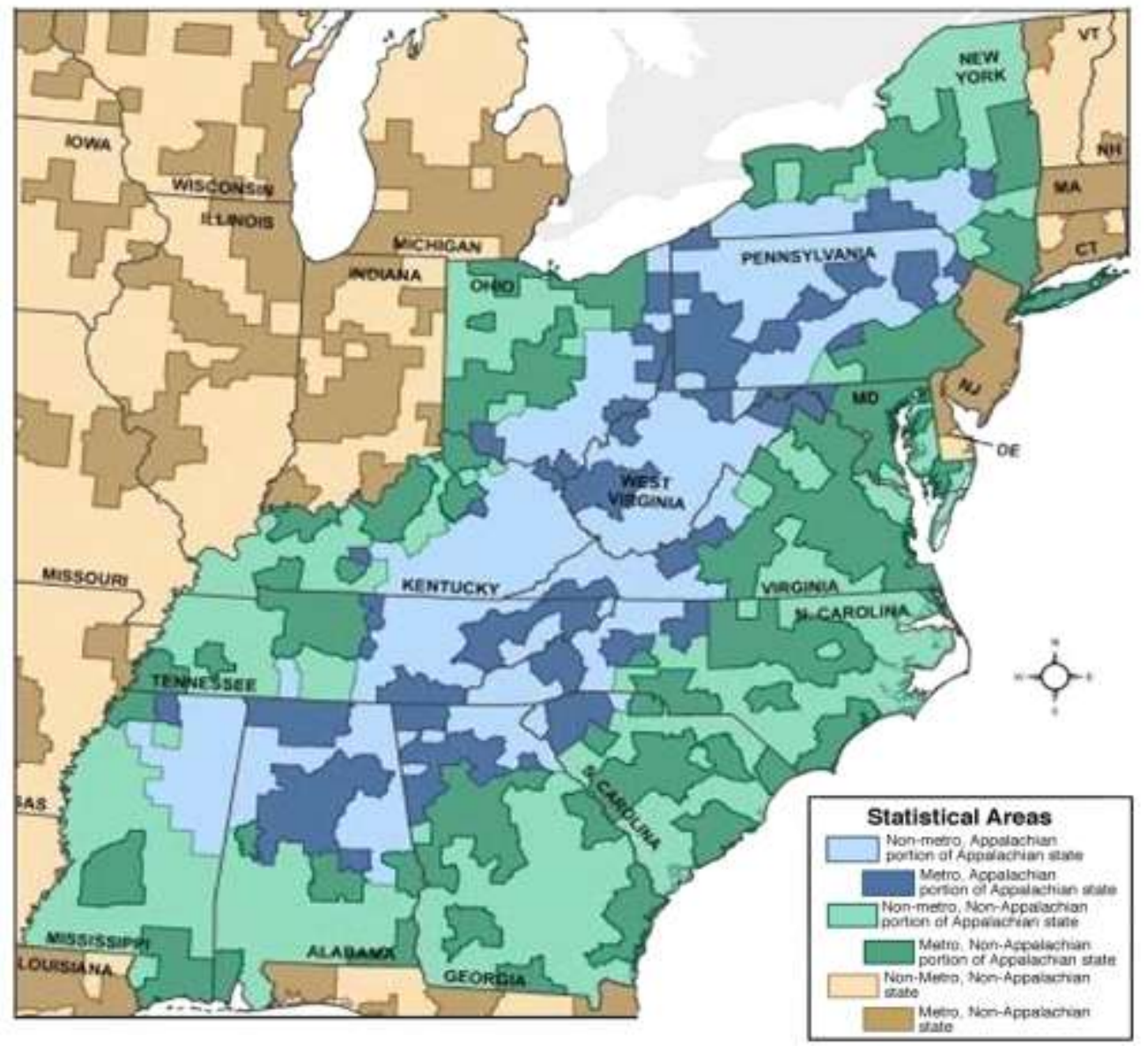

Figure 4. Metropolitan and non-metropolitan areas defined using Beale codes. 
All counties in West Virginia are within the Appalachian region. Since West Virginia data did not meet county-level minimum requirements, West Virginia has prevalence estimates only for metropolitan and non-metropolitan regions. Other states have both Appalachian and non-Appalachian counties. After aggregating and weighting the data to the larger geographic areas just described, we were able to obtain valid $(\mathrm{N} \geq 50)$ contiguous data, and oral health indicators could be mapped and compared to national averages (Figure 5).

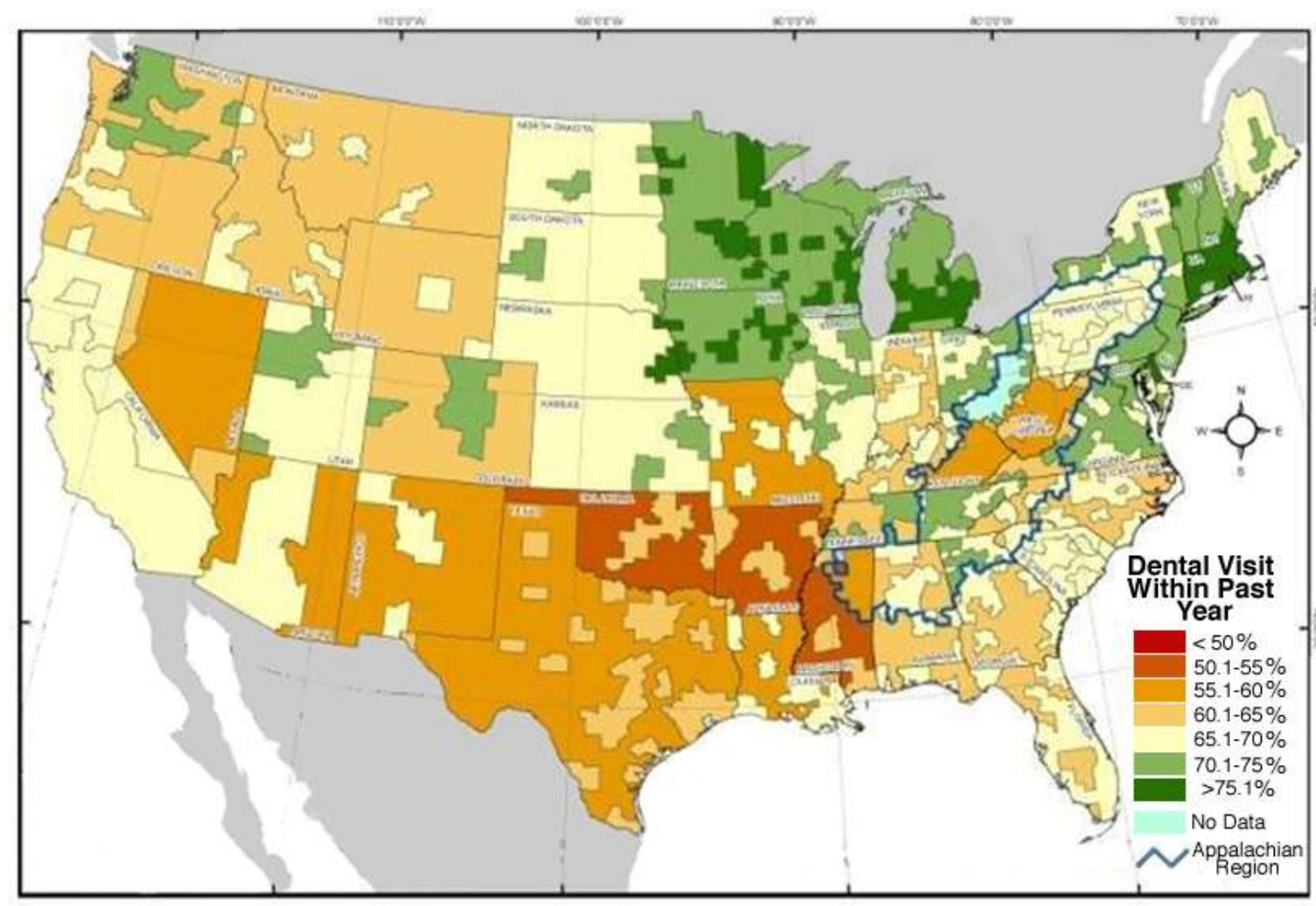

Figure 5. BRFSS data aggregated to metropolitan and non-metropolitan areas defined by Beale codes, showing the proportion of respondents who had a dental visit within the last year for all U.S. counties, with the Appalachian region outlined in blue.

\section{Results}

Figure 1 shows the more typical state-level presentation of BRFSS data, a graphical view of the percentage of persons who had a dental visit within the past year for all states. Of the Appalachian states, Mississippi had the lowest percentage of dental visits, followed by West Virginia, and Kentucky. However, the presentation of BRFSS data at the state-level provides no meaningful differences on sub-state differences.

After combining multiple years of BRFSS data, we mapped the oral health indicators at the county level. Figure 2 illustrates the geographic distribution of dental visits as an oral health indicator for only those counties with sample sizes large enough to meet the CDC guidelines. There was a tremendous amount of missing data and it was clearly not possible to conduct this 
study at the county level, with such sparse data. We did not have enough data for about half of the counties of Appalachia.

We then aggregated those counties that did not have the needed 50 or more responses into larger areas of Appalachia/non-Appalachian portions of the states. Figure 3 provides a more detailed picture of the Appalachian region. At this point, again we see that Mississippi, eastern Kentucky, and West Virginia were areas of greatest concern, with one large county in north Mississippi and two counties in West Virginia especially standing out.

To make the analysis easier to interpret and more informative, we decided to make rural $v s$. urban distinctions, further refining the county-level maps by aggregating the counties into metropolitan and non-metropolitan areas, using Beale codes. Thus, we created four groups for analysis: (1) Appalachian/metropolitan, (2) Appalachian/non-metropolitan, (3) nonAppalachian/metropolitan, and (4) non-Appalachian/non-metropolitan. States that surround the Appalachian region were also mapped for comparisons (Figure 4).

Figure 5 shows the utility of mapping metropolitan/non-metropolitan areas in helping to smooth the oral health data. Regions of Mississippi, eastern Kentucky and West Virginia, had low rates of dental visits, but, now, using Beale codes, we can also see that most of the areas with poorer oral health status (as we measure it), were non-metropolitan areas. Furthermore, other areas within the Appalachian region showed rates similar to the rest of the 48 contiguous United States. The map also reveals apparent differences between the Appalachian region of Mississippi in the northeast corner of the state, with slightly higher rates of dental visits, and the central and Delta regions of the state. Note that Mississippi, Arkansas and Okalahoma had large nonmetropolitan areas where dental visits were at a lower rate than metropolitan areas, a potential indicator of lack of access to care.

\section{Discussion and Limitations}

Oral health data are difficult to obtain for small area estimation. While a county-level analysis of oral health indicators would have been ideal, it proved impossible due to limitations with the availability and accessibility of oral health data. The BRFSS is an extensive and large national dataset that includes oral health questions. However, we found that there were not enough responses to perform county-level analysis for oral health indicators even after combining multiple years of BRFSS data. Unfortunately, the oral health questions are optional and, therefore, are not asked every year in every state. Ultimately, to perform sub-state analyses, we aggregated Appalachian vs. non-Appalachian regions within Appalachian states. Then, using Beale codes, we also examined metropolitan and non-metropolitan differences, while adjusting for poverty. Using BRFSS data, we were able to make estimations smaller than the state level, but not as small as the county or local level, which would have been preferable. There is wide variation in health status throughout the Appalachian region and we were not able to detect local differences using BRFSS data. However, by using the method described, we were able to conduct informative analyses about oral health in the Appalachian region at this sub-state level, examining metropolitan/non-metropolitan differences, and to make further comparisons between Appalachia and the rest of the nation. ${ }^{6}$ 
There is a pressing need for better oral health data to study oral health status and to measure the effectiveness of funded initiatives, nationally, and specifically in the Appalachian region. The BRFSS is a valuable source of data, but it has its limitations for small area estimation as we have shown here. Discussions should begin with The Centers for Disease Control (CDC) to modify the sampling procedures used for the BRFSS to collect consistent responses to oral health questions each year in all states. A larger sampling frame would provide the data needed to better inform public health planners and politicians of what areas or population groups should be targeted to improve oral health conditions in Appalachia.

\title{
Conclusion
}

Aggregating the data as described proved useful for analyzing oral health disparities in Appalachia, while still revealing important sub-state differences. Using GIS to map data provided very useful "views" of the data that helped determine the best methodological approach for conducting this study and provided interesting visual results of the comparisons of oral health indicators in Appalachia. Mapping the data also helped visualize that low socioeconomic status and rurality contribute to oral health disparities in Appalachia.

Figures 1-5 illustrate the process we used to glean meaningful information from limited oral health data. Maps are presented of only one of the oral health indicators obtained from the BRFSS and used for our analysis of oral health disparities in Appalachia. We began the process with BRFSS data intended for state use (Figure 1) and ended up with four groups for analysis. The oral health variable, dental visit within the past year, is presented in Figure 5, showing differences in metropolitan/non-metropolitan areas.

The BRFSS can be a useful data source for studying a number of health topics, but may require some small area estimation techniques such as those described here, to overcome data challenges, especially on health topics not included as core questions in the BRFSS Survey. Using BRFSS health data and these methods to examine oral health disparities in Appalachia, we were able to to make some interesting observations about oral health disparities in Appalachia for policymakers and health planners, identifying areas of concern for targeted intervention strategies.

\section{Corresponding Author}

\author{
Denise D. Krause \\ Associate Professor \\ University of Mississippi Medical Center \\ Email:dkrause@umc.edu
}




\section{References}

1. Satcher D. Oral Health in America: A Report of the Surgeon General. In: U.S. Department of Health and Human Services, editor. Rockville, MD: National Institute of Dental and Craniofacial Research, National Institutes of Health; 2000.

2. Brown LJ, Wall TP, Lazar V. Trends in untreated caries in permanent teeth of children 6 to 18 years old. J Am Dent Assoc. 1999;130(11):1637-44 contd. Epub 1999/11/26. PubMed PMID: 10573947.

3. Newacheck PW, Hughes DC, Hung YY, Wong S, Stoddard JJ. 2000. The unmet health needs of America's children. Pediatrics. 105(4 Pt 2), 989-97. Epub 042000.

4. Mouradian WE, Wehr E, Crall JJ. 2000. Disparities in children's oral health and access to dental care. JAMA. 284(20), 2625-31. Epub 11 2000. http://dx.doi.org/10.1001/jama.284.20.2625 5. Edelstein BL. Disparities in oral health and access to care: findings of national surveys. Ambulatory pediatrics : the official journal of the Ambulatory Pediatric Association. 2002;2(2 Suppl):141-7. Epub 2002/04/13. PubMed PMID: 11950385.

6. Krause DD, May WL, Lane NM, Cossman JS, Konrad TR. An Analysis of Oral Health Disparities and Access to Services in the Appalachian Region. In: Appalachian Regional Commission, editor. Washington, D.C. 2012.

7. Jia H, Muennig P, Borawski E. 2004. Comparison of small-area analysis techniques for estimating county-level outcomes. Am J Prev Med. 26(5), 453-60. Epub 05 2004. doi:http:// dx.doi.org/10.1016/j.amepre.2004.02.004.

8. Jia H, Link M, Holt J, Mokdad AH, Li L, et al. 2006. Monitoring county-level vaccination coverage during the 2004-2005 influenza season. Am J Prev Med. 31(4), 275-80. Epub 092006. doi:http://dx.doi.org/10.1016/j.amepre.2006.06.005.

9. Congdon P. 2009. A multilevel model for cardiovascular disease prevalence in the US and its application to micro area prevalence estimates. Int J Health Geogr. 8, 6. Epub 02 2009. doi:http:// dx.doi.org/10.1186/1476-072X-8-6.

10. Li W, Land T, Zhang Z, Keithly L, Kelsey JL. 2009. Small-area estimation and prioritizing communities for tobacco control efforts in Massachusetts. Am J Public Health. 99(3), 470-79. Epub 01 2009. doi:http://dx.doi.org/10.2105/AJPH.2007.130112.

11. Schneider KL, Lapane KL, Clark MA, Rakowski W. 2009. Using small-area estimation to describe county-level disparities in mammography. Prev Chronic Dis. 6(4), A125. Epub 092009. 12. Zhang Z, Zhang L, Penman A, May W. 2011. Using small-area estimation method to calculate county-level prevalence of obesity in Mississippi, 2007-2009. Prev Chronic Dis. 8(4), A85. Epub 062011.

13. Office of Surveillance, Epidemiology, and Laboratory Services. Questionnaires: Centers for Disease Control and Prevention; 2011 [cited 2012 10/10/2012]. Available from: http:// www.cdc.gov/brfss/questionnaires/index.htm.

14. Office of Surveillance, Epidemiology, and Laboratory Services. BRFSS Annual Survey Data: Centers for Disease Control and Prevention; 2012 [11/27/2012]. Available from: http:// www.cdc.gov/brfss/technical infodata/weighting.htm.

15. Economic Research Service. Rural-Urban Continuum Codes: United States Department of Agriculture; 2004 [updated November 3, 2004April 18, 2012]. Available from: http:// www.ers.usda.gov/Data/RuralUrbanContinuumCodes/. 
